\title{
Effect of Safflower Oil on the Protective Properties of the in situ Formed Salivary Pellicle
}

\author{
C. Hannig ${ }^{\text {a }} \quad$ C. Wagenschwanz ${ }^{\text {a }} \quad$ S. Pötschke ${ }^{\text {a }} \quad$ K. Kümmerer ${ }^{\text {b }} \quad$ A. Kensche ${ }^{\text {a }}$ \\ W. Hoth-Hannig ${ }^{c}$ M. Hannig ${ }^{c}$ \\ ${ }^{a}$ Clinic of Operative Dentistry, Medical Faculty Carl Gustav Carus, TU Dresden, Dresden, ${ }^{\mathrm{b}}$ Material Resources, \\ Institute for Environmental Chemistry, Leuphana University Lüneburg, Lüneburg, and ' ${ }^{~ C l i n i c ~ o f ~ O p e r a t i v e ~ D e n t i s t r y, ~}$ \\ Periodontology and Preventive Dentistry, University Hospital, Saarland University, Homburg/Saar, Germany
}

\section{Key Words}

Edible oil $\cdot$ Erosion $\cdot$ In situ formed pellicle $\cdot$ Lipids

\begin{abstract}
Aim: The prevalence of dental erosion is still increasing. A possible preventive approach might be rinsing with edible oils to improve the protective properties of the pellicle layer. This was tested in the present in situ study using safflower oil. Methods: Pellicle formation was carried out in situ on bovine enamel slabs fixed buccally to individual upper jaw splints (6 subjects). After 1 min of pellicle formation subjects rinsed with safflower oil for $10 \mathrm{~min}$, subsequently the samples were exposed in the oral cavity for another $19 \mathrm{~min}$. Enamel slabs without oral exposure and slabs exposed to the oral cavity for $30 \mathrm{~min}$ without any rinse served as controls. After pellicle formation in situ, slabs were incubated in $\mathrm{HCl}$ $(\mathrm{pH} 2 ; 2.3 ; 3)$ for $120 \mathrm{~s}$, and kinetics of calcium and phosphate release were measured photometrically (arsenazo III, malachite green). Furthermore, the ultrastructure of the pellicles was evaluated by transmission electron microscopy (TEM). Results: Pellicle alone reduced erosive calcium and phosphate release significantly at all $\mathrm{pH}$ values. Pellicle modification by safflower oil resulted in an enhanced calcium loss at
\end{abstract}

all $\mathrm{pH}$ values and caused an enhanced phosphate loss at $\mathrm{pH}$ 2.3. TEM indicated scattered accumulation of lipid micelles and irregular vesicle-like structures attached to the oil-treated pellicle layer. Acid etching affected the ultrastructure of the pellicle irrespective of oil rinsing. Conclusion: The protective properties of the pellicle layer against extensive erosive attacks are limited and mainly determined by $\mathrm{pH}$. The protective effects are modified and reduced by rinses with safflower oil.

Copyright $\odot 2012$ S. Karger AG, Basel

The prevalence of dental erosion is still increasing as shown in many epidemiological studies [Larsen et al., 2005; Jaeggi and Lussi, 2006]. Stages from initial tooth surface alterations with microscopically detectable enamel loss to more than $50 \%$ of tooth surface loss can be found [Lussi et al., 2006]. Demineralization is induced by intrinsic or extrinsic acids. Main causes of erosion are different dietary substances containing acids, especially the extensive consumption of acidic beverages by vast parts of the population [Lussi et al., 2004]. The erosive effects of acidic foodstuffs or beverages suggest that dietary additives providing $\mathrm{pH}$-buffering effects could be one ap-

\section{KARGER \\ Fax +4161306 1234 \\ E-Mail karger@karger.ch}

www.karger.com (c) 2012 S. Karger AG, Basel

$0008-6568 / 12 / 0465-0496 \$ 38.00 / 0$

Accessible online at:

www.karger.com/cre
Christian Hannig

Clinic of Operative Dentistry, Medical Faculty Carl Gustav Carus

TU Dresden, Fetscherstrasse 74

DE-01307 Dresden (Germany)

Tel.+49351 458 2713, E-Mail christian.hannig@uniklinikum-dresden.de 
proach for the prevention or reduction of erosive mineral loss, e.g. calcium or milk derivatives such as casein phosphopeptides could be added to orange juice [Reynolds, 1998; Hannig and Hannig, 2010; Wegehaupt et al., 2011]. Moreover, fluoridated toothpaste has been shown to provide a protective effect against enamel erosion, if used regularly [Magalhaes et al., 2008]. Furthermore, in different culture groups mouth rinsing or oil pulling with edible oils is already part of body care. During oil pulling, a tablespoon of edible oil is taken into the mouth, sucked, sipped and pulled between the teeth over minutes, sometimes till the viscous oil turns thin and foamy. It is assumed that this procedure helps to remove bacteria from the oral cavity [Asokan, 2008; Asokan et al., 2011]. Typical examples for oil pulling agents are olive oil, sesame oil, safflower oil or linseed oil. Oil pulling could also mean an alternative approach to prevent erosive damage by adding more hydrophobic properties to the tooth surface due to accumulation of lipids in the acquired pellicle layer. The pellicle formed on all solid substrates exposed to the oral fluids is regarded as one key factor in the physiological prevention of dental erosion [Hannig and Joiner, 2006]; it has been shown that lipids account for $22-23 \%$ of the dry weight of the pellicle [Slomiany et al., 1986, 1990]. Though knowledge on lipids in the acquired pellicle is based on only these two publications [Slomiany et al., 1986, 1990], their possible impact on the protective properties has been discussed extensively [Hannig and Joiner, 2006]. Furthermore, several in vitro studies neglecting the in situ pellicle indicated that vegetable oil mouthrinses or olive oil-containing preparations might form a protective coating on dental hard tissues decreasing erosive mineral loss [Buchalla et al., 2003; Wiegand et al., 2007]. Irrespective of the lipid composition the pellicle is regarded as a semi-permeable barrier and buffer reducing erosive mineral loss [Hara et al., 2006; Hannig et al., 2009]. Due to its mesh-like ultrastructure, it provides a medium through which ions such as fluoride, calcium or phosphate are delivered during de- and re-mineralization [Schüpbach et al., 2001; Hannig, 2002; Hannig and Joiner, 2006]. Nevertheless, the protective properties are limited and the ultrastructure of the pellicle is degraded continuously at low pH levels [Hannig and Balz, 1999, 2001; Hannig et al., 2003, 2009].

Many edible oils have a pronounced particular flavor, whereas safflower oil is rather tasteless. There are two types of safflower oil which are regarded as beneficial in preventive medicine. One type is rather rich in monounsaturated oleic acid, the other in linoleic acid [Krist et al., 2008]. The high concentration of unsaturated fatty acids, especially linoleic acid, is considered to be valuable for the reduction of cholesterol levels [Czernichow et al., 2010; Higa et al., 2010; Suzuki et al., 2010]. Due to the dominance of essential $\omega-6$ fatty acids, safflower oil was also used as a component of parenteral nutrition solutions. The aim of the present in situ/in vitro study was to evaluate the effect of safflower oil on the protective properties of the in situ formed pellicle. For this purpose, pellicles formed in situ on bovine enamel slabs and modified by rinses with safflower oil were incubated in hydrochloric acid in vitro. Thereby, the study focussed on short-term erosive effects simulating the situation in the oral cavity. The erosive effects were measured via determination of calcium and phosphate release. In addition, transmission electron microscopic investigations were performed to visualize the impact of rinsing with oil on pellicle ultrastructure in an acidic milieu.

\section{Methods}

Subjects

A number of 6 healthy volunteers, members of the laboratory staff (age 24-42), participated in the study. Visual oral examination was carried out by an experienced dentist. The subjects showed no signs of gingivitis or caries; they had a physiological salivary flow rate as determined qualitatively during oral examination. Informed written consent had been given by the subjects about participation in the study. The study design was reviewed and approved by the Ethics Committee of the University of Freiburg in Germany (Proposal 222/08).

\section{Specimen Preparation}

Enamel slabs with a diameter of $5 \mathrm{~mm}$ were prepared from bovine incisor teeth (2-year-old cattle). In total, 398 slabs were prepared. According to the size of the teeth, between 2 and 3 samples were gained from each bovine incisor. Specimens were etched at all sites except for the outer enamel surface for $15 \mathrm{~s}$ with $37 \%$ phosphoric acid gel (Etching gel, DMG, Hamburg, Germany) and treated for $30 \mathrm{~s}$ with Optibond Primer (Kerr, Karlsruhe, Germany). Optibond Adhesive was applied afterwards and light-cured for $30 \mathrm{~s}$. Application of the adhesive was performed 3 times. Subsequently, the unsealed enamel surfaces were ground flat and polished (grit 4000) [Hannig et al., 2007a]. In a standardized grinding procedure, approximately $200 \mu \mathrm{m}$ of the enamel was removed. Specimens with structural alterations of the enamel were excluded from the study. The smear layer on the slabs was removed with a steam jet and by ultrasound with $\mathrm{NaOCl}$ for 3 min [Hannig et al., 2007b]. Then the slabs were washed twice in distilled water for $5 \mathrm{~min}$ also by ultrasound. Afterwards, the samples were disinfected in ethanol (70\%) for another $10 \mathrm{~min}$ (ultrasound), washed in distilled water and stored in distilled water for $24 \mathrm{~h}$ before exposure in the oral cavity.

Different experimental setups were tested to evaluate the effect of rinsing with safflower oil on the acquired pellicle and on erosive mineral loss (fig. 1). 


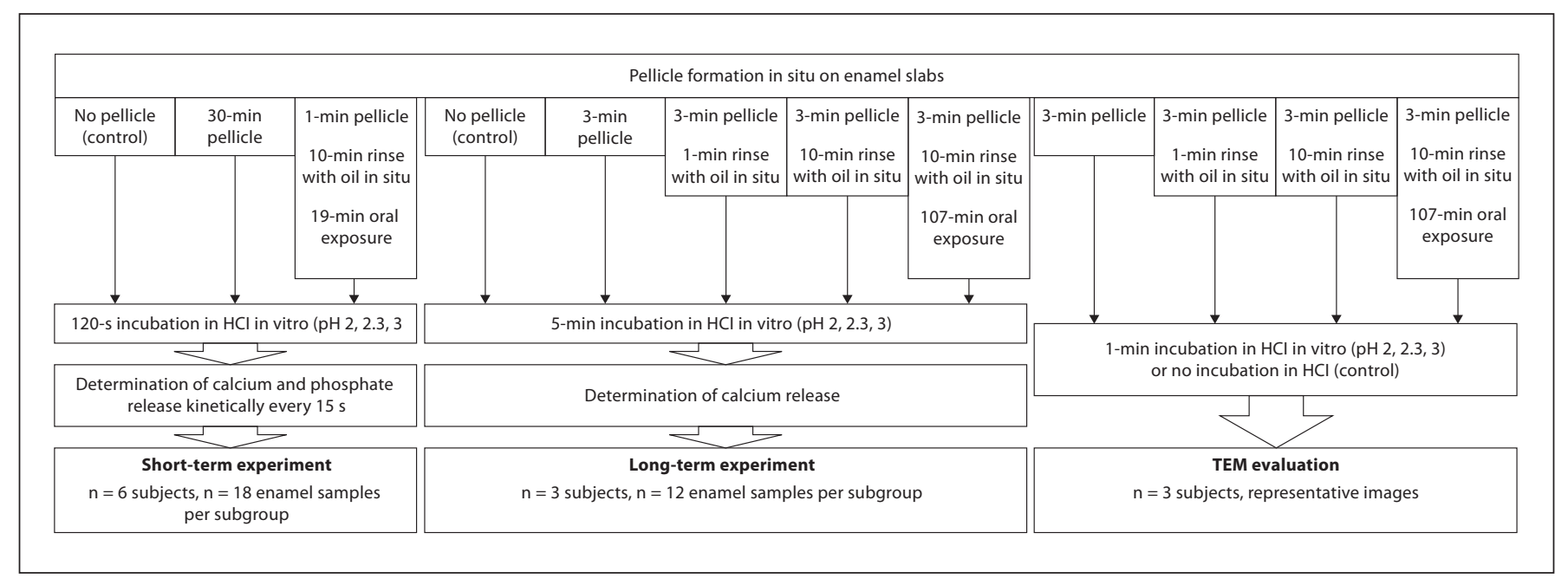

Fig. 1. Flow chart of all experiments.

\section{In situ Pellicle Formation and in vitro Erosion}

For in situ pellicle formation, individual upper jaw splints were vacuum-formed from 1.5-mm-thick methacrylate foil for each volunteer. Cavities were prepared in the buccal aspects of the splints (in the region of $16,15,14$, and the region of $24,25,26$, respectively). A number of 6 slabs were fixed on each splint with polyvinylsiloxane impression material (President Light Body, Coltène, Switzerland) in such a way, that only the specimen's surfaces were exposed to the oral environment. The splints were carried intraorally for $1 \mathrm{~min}$ to allow initial pellicle formation on the specimen surfaces. Afterwards, the subjects rinsed thoroughly with $8 \mathrm{ml}$ safflower oil (Brölio Distelöl, Brökelmann \& Co. Ölmühle GmbH \& Co., Hamm, Germany) for $10 \mathrm{~min}$. The splints remained in the oral cavity for further $19 \mathrm{~min}$; this regime gave a total intraoral exposure time of $30 \mathrm{~min}$. After intraoral exposure, the slabs were quickly removed from the splints and rinsed thoroughly with running water for $5 \mathrm{~s}$. In vitro erosion was performed by incubating each sample in $1,000 \mu$ hydrochloric acid $(\mathrm{pH} 2$, $2.3,3)$ to provide an excess of acid in order to maintain constant $\mathrm{pH}$ during the incubation period of $120 \mathrm{~s}$ (short-term experiment, fig. 1). Before exposure to $\mathrm{HCl}$, the enamel slabs were embedded in silicone impression material at the bottom of a 2-ml Eppendorf cup. The acid was moved continuously by pumping with a $100-\mu \mathrm{l}$ pipette (1 lift/s). Every $15 \mathrm{~s}, 100 \mu \mathrm{l}$ of the acid was removed for photometric analysis and replaced by $100 \mu \mathrm{l}$ of fresh acid. For control purposes, enamel specimens coated with a 30 -min salivary pellicle (without oil rinsing) and specimens without pellicle were incubated in $\mathrm{HCl}$.

Furthermore, calcium loss after 5-min incubation in $\mathrm{HCl}$ was measured in order to investigate the protective effect of the pellicle during longer incubation in an acidic agent (long-term experiment, fig. 1). In this additional experiment the following pretreatments of the enamel slabs under in situ conditions were tested: no pellicle; 3 -min pellicle formation followed by 1-min rinse with oil; 3-min pellicle followed by 10-min rinse with oil and 107min further oral exposure in order to have a total intraoral expo- sure time of $120 \mathrm{~min}$ (120-min pellicle); 3 -min pellicle; 3 -min pellicle followed by 10 -min rinse with oil. Three subjects took part in this additional experiment, each subject carried 4 samples per subgroup in the oral cavity (12 enamel samples).

\section{Photometric Determination of Calcium and Phosphate \\ Release}

Mineral dissolution caused during incubation in $\mathrm{HCl}$ was determined by measuring photometrically calcium and phosphate release into the solution in double assays using the arsenazo III method (Fluitest ${ }^{\circledR}$, Ca-A-II, Analyticon, Lichtenfels, Germany) and the malachite green assay, as described previously [Attin et al., 2005a, b; Hannig et al., 2005, 2007a].

Arsenazo III reacts with calcium in an acidic solution to form a blue purple complex. The resulting intensity is proportional to the calcium concentration. Absorption can be determined at $\lambda=$ $650 \mathrm{~nm}$ according to standard curves. The reagent for determination of calcium was composed of $100 \mathrm{~mm}$ imidazole buffer $(\mathrm{pH}$ 6.5) and $0.12 \mathrm{mM}$ arsenazo III. A volume of $10 \mu \mathrm{l}$ from the sample was pipetted to $100 \mu \mathrm{l}$ arsenazo reagent [Hannig et al., 2005, 2007a]. Two samples were measured for each specimen, and the average absorption was calculated.

Malachite green reacts with phosphate to form a colored complex which can be determined photometrically at $\lambda=650 \mathrm{~nm}$. For the test reagent $0.045 \mathrm{mg}$ of malachite green dissolved in $100 \mathrm{ml}$ distilled water was mixed with $12.69 \mathrm{~g}$ of ammonium molybdate dissolved in $300 \mathrm{ml} \mathrm{HCl}(4 \mathrm{M})$. The reagent was stirred for $30 \mathrm{~min}$ afterwards and filtered (pore size $0.22 \mu \mathrm{m}$ ).

A volume of $10 \mu \mathrm{l}$ from the sample was pipetted to $200 \mu \mathrm{l}$ malachite reagent; the absorption was measured after $15 \mathrm{~min}$ [Attin et al., 2005b]. Two samples were measured for each specimen, and the average absorption was calculated. Calcium and phosphate release were calculated based on the mean photometric absorption values for the specimens and their surface areas (based on $5 \mathrm{~mm}$ diameter). The assays were also used for determination of calcium and phosphate concentrations of the saliva and of the oil. 
Transmission Electron Microscopy (fig. 1)

In addition, in situ pellicle samples were prepared for electron microscopic analysis in order to visualize the impact of rinses with safflower oil on the ultrastructure of the pellicle and on its protective properties. Pellicle formation was performed for $3 \mathrm{~min}$ followed by a $10-\mathrm{min}$ rinse with safflower oil. Then the samples were removed from the splints and transferred to the in vitro experiments, or carried in the oral cavity for another $107 \mathrm{~min}$ in order to have a total intraoral exposure time of $120 \mathrm{~min}$. The samples were incubated in $\mathrm{HCl}(\mathrm{pH} 2 ; 2.3,3)$ in vitro for $1 \mathrm{~min}$, or prepared for transmission electron microscopy (TEM) without incubation in $\mathrm{HCl}$. Enamel samples with 3 -min pellicles and 120 -min pellicles, but without rinsing with safflower oil, served as controls. Immediately after the erosion experiments, the enamel slabs were fixed in glutaraldehyde for $2 \mathrm{~h}$ (2.5\% glutaraldehyde, 1.5\% formaldehyde in phosphate buffer, $\mathrm{pH}$ 7.4). Afterwards, the specimens were washed 5 times in phosphate buffer. Post-fixation for visualization of organic structures took place in $1 \%$ osmium tetroxide for $2 \mathrm{~h}$. The specimens were dehydrated in increasing concentrations of alcohol and embedded in Araldite M (Serva, Darmstadt, Germany). The dentine was removed from the samples with a diamond bur, and the samples were decalcified in $1 \mathrm{M} \mathrm{HCl}$. Re-embedding was performed with Araldite. Ultrathin sections of the pellicle samples were cut in series with an ultramicrotome (Ultracut E, Reichert, Bensheim, Germany), using a diamond knife. The ultrathin sections were mounted on mesh grids (Plano, Wetzlar, Germany) and contrasted with uranyl acetate and lead citrate. TEM investigation took place at 3,000- to 50,000-fold magnification in a TEM TECNAI 12 Biotwin (FEI, Eindhoven, The Netherlands).

\section{Statistics}

Data of the short-term experiments were evaluated by one-way ANOVA followed by the Scheffé procedure in order to detect significant influences of pellicle formation and safflower oil-rinsing on calcium and phosphate loss during incubation in $\mathrm{HCl}$. Statistical analysis was performed considering the data from the 18 enamel slabs from all 6 subjects of each experimental subgroup for calculation of the means that were compared for significant differences. Normal distribution was checked using the Kolmogorov-Smirnov test; the software used was SPSS 17.0 (StatSoft, Hamburg, Germany). The level of significance was set at $\mathrm{p}<0.05$.

\section{Results}

\section{Photometric Determination of Calcium and} Phosphate Release

In general, the erosive mineral loss was predominantly determined by the $\mathrm{pH}$ of the hydrochloric acid, irrespective of whether the pellicle was modified or not by rinsing with safflower oil (fig. 2, 3, table 1). The kinetics of calcium and phosphate release over $120 \mathrm{~s}$ were almost linear irrespective of the pellicle layer or its modification by rinsing with oil. However, the 30 -min non-rinsed pellicle reduced the erosive mineral loss at all $\mathrm{pH}$ values.

Due to the linearity of calcium and phosphate release, statistical evaluation was conducted for the cumulative
120 -second data. During exposure in $\mathrm{HCl}$ for $120 \mathrm{~s}$, calcium release was significantly reduced by the 30 -min pellicle layer at pH 2.0 (-20\%), pH $2.3(-25 \%)$, and $\mathrm{pH} 3.0$ $(-64 \%)$ compared with the controls without pellicle (table 1). Rinsing with safflower oil led to a slightly higher loss of calcium from the pellicle-covered specimens than from slabs with 30 -min non-rinsed pellicles at all $\mathrm{pH}$ values $(\mathrm{pH} 3.0$ : $\mathrm{p}<0.05)$.

In part, divergent effects were observed for the kinetics of phosphate release recorded over $120 \mathrm{~s}$. At all pH values the physiological 30 -min pellicle (without oil rinsing) reduced the erosive phosphate loss significantly $(\mathrm{pH}$ 2: $-25 \%, \mathrm{pH} 2.3:-45 \%, \mathrm{pH} 3:-41 \%)$. In contrast to the kinetics of calcium release, the results for phosphate indicate that rinsing with safflower oil did not significantly impair the protective effect of the 30-min non-rinsed pellicle at $\mathrm{pH} 2.0$ and 3.0. However, at $\mathrm{pH} 2.3$, a significantly higher phosphate dissolution from enamel samples covered by an in situ pellicle modified by a rinse with safflower oil was observed compared to the 30-min nonrinsed pellicle $(\mathrm{p}<0.05)$.

In contrast to the results gained after 120 -second $\mathrm{HCl}$ exposure, application of safflower oil in situ had no inhibiting influence on the cumulative erosive mineral loss after 5-min exposure to $\mathrm{HCl}$ (fig. 4). Thereby, different rinsing modes were tested without observing any significant effect.

The calcium and phosphate concentration of the safflower oil were very low: calcium concentration $=1.61 \times$ $10^{4} \mathrm{nM}$; phosphate $=3.95 \times 10^{4} \mathrm{nM}$. The calcium concentration in the saliva ranged between 1 and $1.5 \times$ $10^{6} \mathrm{nM}$, the phosphate concentration between 2.5 and $3 \times 10^{6} \mathrm{nM}$.

\section{Transmission Electron Microscopy}

The TEM images gave additional insight into the interaction of safflower oil with the pellicle layer; the effect of $\mathrm{HCl}$ on physiological and modified pellicles was visualized (fig. 5, 6). Rinses with oil for $10 \mathrm{~min}$ induced ultrastructural alterations of the 3-min pellicle layer (fig. 5). Small oil micelles (200-400 $\mathrm{nm}$ in diameter) were detected on the surface of the pellicle. However, the principal structure of the basal pellicle was not changed, large areas of the pellicle looked like typical physiological 3 -min pellicles. If the samples were carried in the oral cavity for another $107 \mathrm{~min}$ (120-min pellicles), after rinsing with safflower oil, for further pellicle formation, a different ultrastructure as compared with the controls was observed. The pellicle was of lower density, including some irregularly shaped vesicle-like structures. 
Fig. 2. Kinetics of calcium loss during incubation of enamel slabs for $120 \mathrm{~s}$ with and without safflower oil rinsing in situ. Physiological 30-min pellicles (without rinse) and specimens without pellicle served as controls. Note the different scale of the experiments performed at $\mathrm{pH} 3 ; \mathrm{n}=6 \mathrm{sub}$ jects, $n=18$ enamel samples per subgroup, mean values \pm standard deviation.

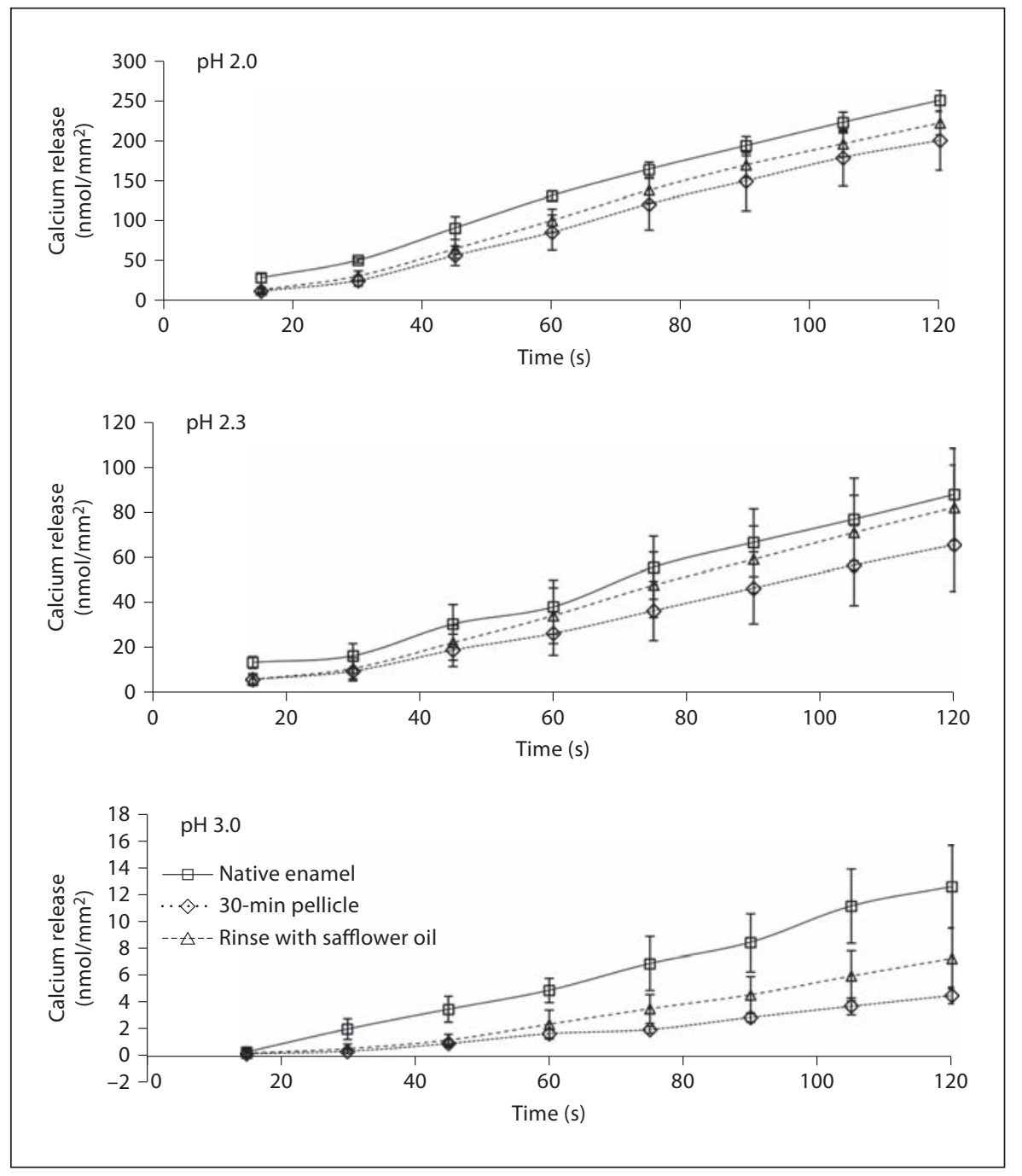

Table 1. Cumulative calcium and phosphate loss $\left(\mathrm{nmol} / \mathrm{mm}^{2}\right)$ from control and test enamel slabs following incubation for $2 \mathrm{~min}$ in $\mathrm{HCl}$ at different $\mathrm{pH}$ values

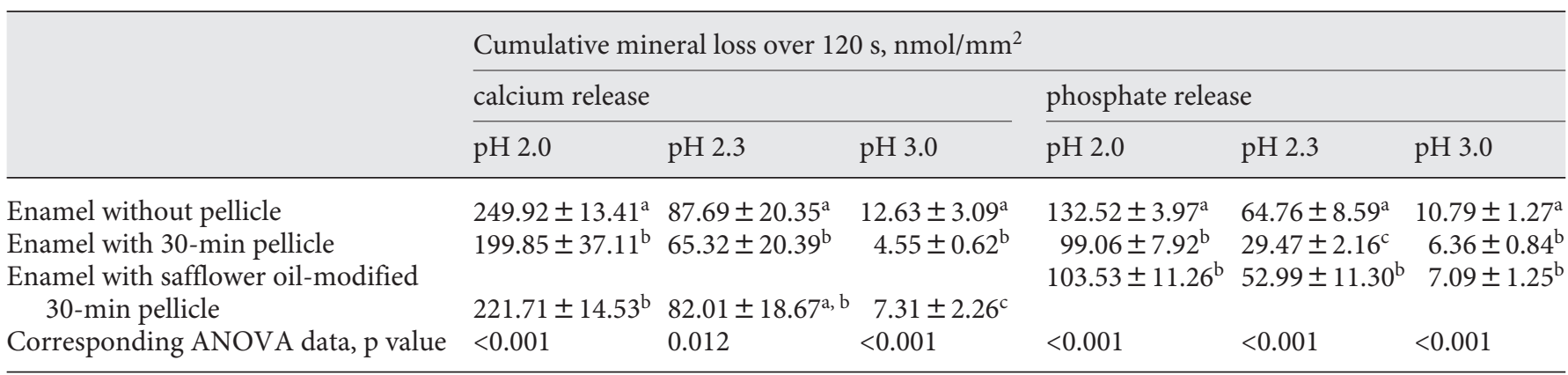

Physiological 30-min pellicles (without oil rinse) and specimens without pellicle served as controls; $\mathrm{n}=6$ subjects, $\mathrm{n}=18$ enamel samples per subgroup. Data significantly different within one column are followed by distinct superscripts ( $\mathrm{p}<0.05$, Scheffé procedure). 
Fig. 3. Kinetics of phosphate loss during incubation of enamel slabs for $120 \mathrm{~s}$ with and without safflower oil rinsing in situ. Physiological 30-min pellicles (without rinse) and specimens without pellicle served as controls. Note the different scale of the experiments performed at $\mathrm{pH} 3 ; \mathrm{n}=$ 6 subjects, $\mathrm{n}=18$ enamel samples per subgroup, mean values \pm standard deviation.

Fig. 4. Incubation of enamel samples for 5 min in $\mathrm{HCl}$ after different modes of pellicle formation in situ with and without safflower oil rinsing; cumulated loss of calcium; $\mathrm{n}=3$ subjects, $\mathrm{n}=12$ enamel samples per subgroup, mean values \pm standard deviation.
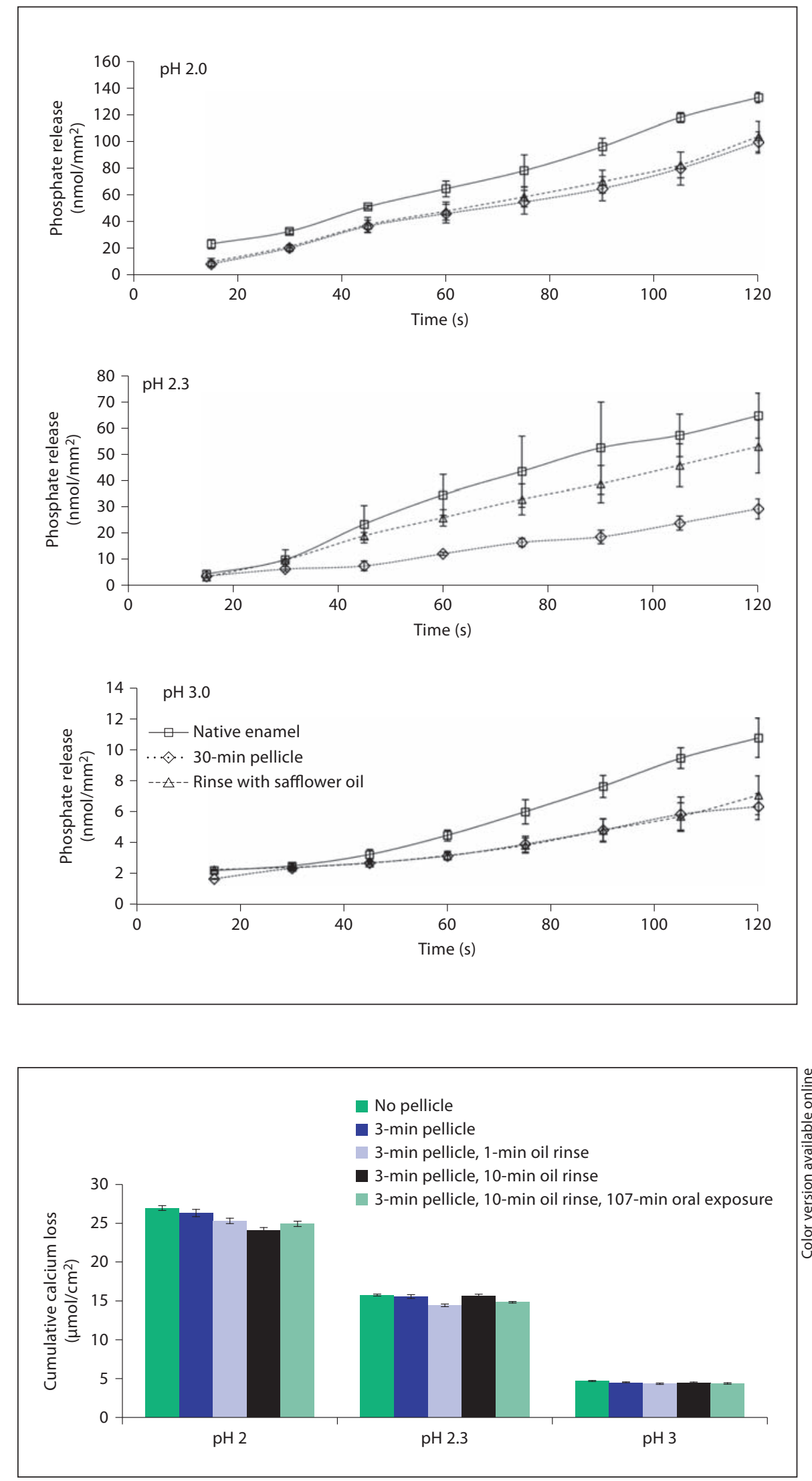
Fig. 5. TEM micrographs of 3-min pellicles (a, d-g) and 120-min pellicles (b, c, $\mathbf{h}, \mathbf{i})$ with and without 10 -min oil rinsing. The 3-min pellicle without oil application showed a fine electron-dense structure (basal pellicle layer, a). Rinses with oil for 10 min mainly yielded a pellicle with a similar basal structure (d), in some cases, small irregular vesicle-like structures were visible (e, arrow). Lipid micelles (arrows) were observed attached randomly to the pellicle layer (f, g). The 120-min pellicle without oil application showed a fine granular structure on top of an electron-dense basal layer (b, c). Rinses with oil for $10 \mathrm{~min}$ after 3-min pellicle formation followed by a 107-min oral exposure (120-min pellicles) yielded a pellicle with a lower density (h, i), sometimes irregular vesicle-like structures are visible (i, triangle). Note that the enamel was removed during the preparation of the samples, the former enamel site is marked with asterisks.

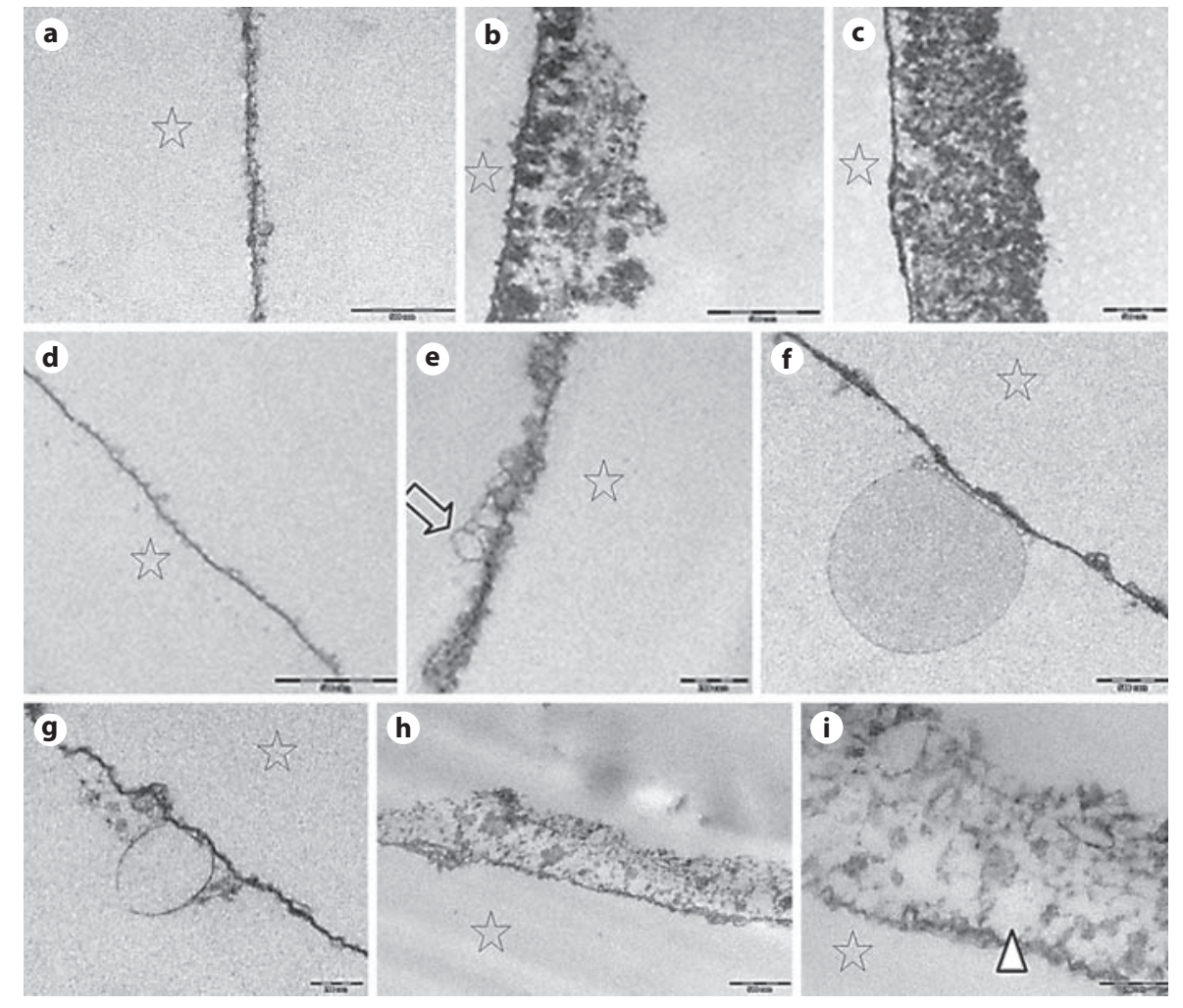

The ultrastructure of the pellicle was degraded during incubation in $\mathrm{HCl}$ in a $\mathrm{pH}$-dependent manner (fig. 6). Thereby, the preliminary rinses with the edible oil had no protective effect on the degradation of the pellicle layer. Sometimes, the degradation process of the proteinaceous layer seemed to be more pronounced in the oil-rinsed specimens. This applied for the 3-min as well as for the 120 -min samples and corresponded to the results of the calcium and phosphate assay.

\section{Discussion}

The present investigation based on in situ formed pellicles monitored short-term erosive effects occurring during the first $5 \mathrm{~min}$ of exposure to acidic agents [Attin, 2006]. Thereby, strong emphasis was put on short-term experiments, measuring the erosive mineral loss from 15 to $120 \mathrm{~s}$ in a kinetic manner. To the best knowledge of the authors, erosive mineral loss from enamel slabs coated with an in situ pellicle has not been investigated in such short intervals until now. The different results observed in the present study within $120 \mathrm{~s}$ and after $5 \mathrm{~min}$ of erosion in $\mathrm{HCl}$ underline the necessity of short-term experiments.
The photometric calcium and phosphate assays are characterized by very high sensitivity and precision [Attin, 2006; Hannig et al., 2008]. Their only disadvantage is that they cannot be used in situ or in vivo [Attin et al., 2005a, b; Hannig et al., 2008]. Accordingly, a combined in situ/in vitro approach was chosen in the present study to ensure formation of physiological (without rinse) and modified (oil-rinsed) in situ pellicles followed by incubation with $\mathrm{HCl}$ in vitro under standardized conditions simulating short-term exposure to acidic agents [Hannig et al., 2007a]; $\mathrm{pH}$ values were chosen according to a previous study on erosive calcium and phosphate release [Hannig et al., 2005].

Several edible oils have been discussed for application in preventive dentistry and preventive medicine [Wiegand et al., 2007; Asokan et al., 2009]. The present study focussed on safflower oil, which is rather tasteless. In contrast to the widely used olive oil, it does not contain many polyphenolic components that will modulate the taste and possibly result in denaturation of the pellicle. Furthermore, it is noteworthy that the mineral content of safflower oil itself is very low and will not affect the erosive process. 


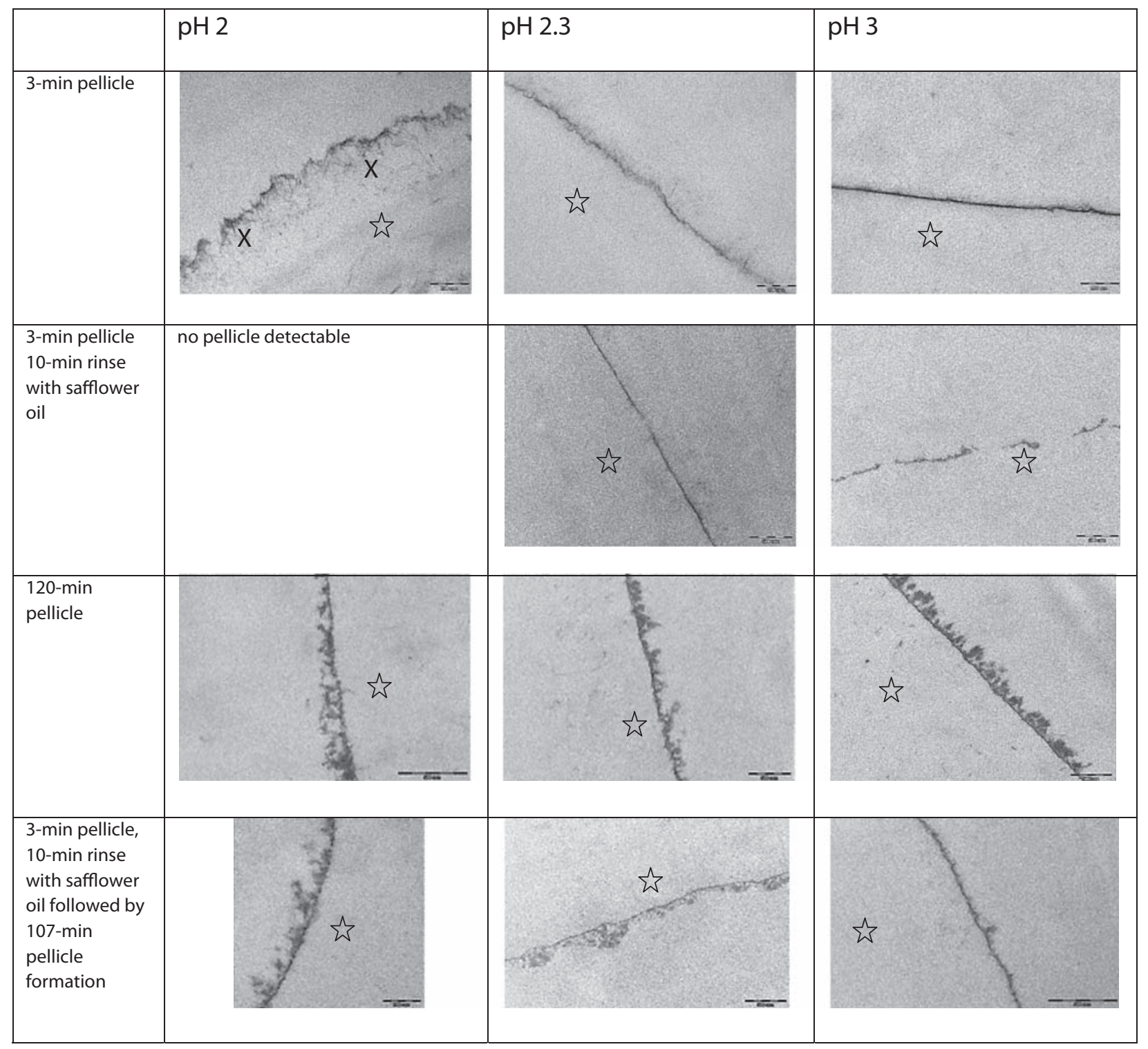

Fig. 6. Acid etching with $\mathrm{HCl}$ for 1 min destroyed the pellicle structure in a time- and $\mathrm{pH}$-dependent manner. No protective effect of the previously performed oil rinsing was visible. Sometimes, the pellicle modified by safflower oil rinsing was degraded in a more pronounced manner than the physiological pellicle (without rinse). At $\mathrm{pH}$ 2, acid etching caused considerable de- struction of the physiological 3-min pellicle (without rinse) and induced extensive erosion refilled with adsorbed proteins $(X)$. Following oil rinsing, no residual pellicle structures were detectable after acid etching at $\mathrm{pH} 2$ in many cases. Note that the enamel was removed during the preparation of the samples, the former enamel sites are marked with asterisks.
In general, the present study showed that rinses with safflower altered calcium and phosphate loss as compared with non-rinsed pellicle samples; in many cases an enhanced mineral loss was recorded. The electron microscopic analysis, though based on 3-min pellicles in contrast to the 1-min specimens adopted in the calcium and phosphate assay, offers an explanation for this phenom- enon. In this context, it should be noted that previous investigations on the ultrastructure of the pellicle clearly indicated that the 1 - and the 3-min pellicle are virtually identical [Hannig, 1999, 2002]. The TEM images clearly indicate that the application of safflower oil did not retard pellicle disintegration under acidic conditions, though accumulation of lipid vesicles at the pellicle sur- 
Fig. 7. Model for the initial stage of pellicle formation. Pellicle precursor proteins interact with a layer of calcium and phosphate ions. In the presence of saliva, the calcium ions of the enamel structure have a stronger tendency to dissolve than the phosphate ions. This leads to a negative charge and results in a layered arrangement of the ions [Hay et al., 1982; Moreno et al., 1982; Hannig and Joiner, 2006]. This will have an impact on the kinetics of mineral loss during the initial phase of an erosive attack. Figure from Hannig and Joiner [2006], modified.

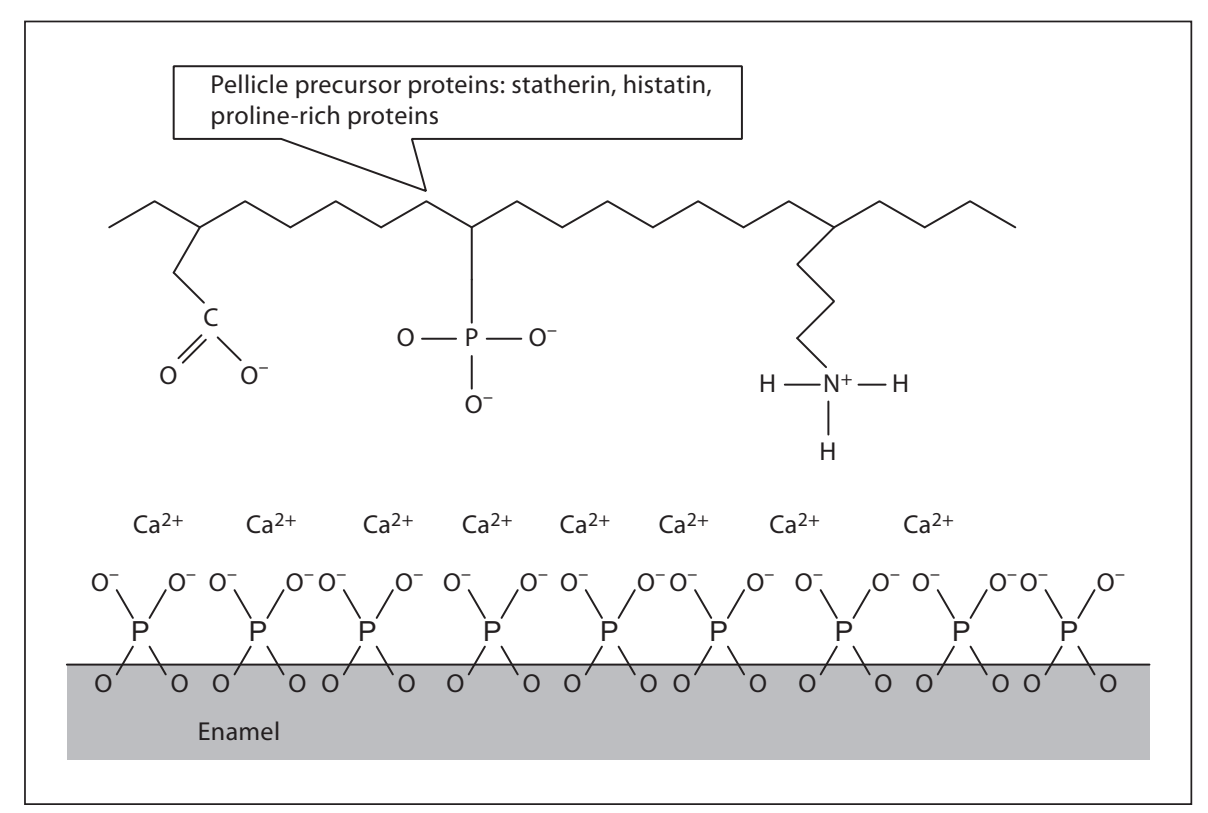

face and an altered ultrastructure were observed after rinsing with safflower oil. The adsorbed lipid micelles caused a scattered accumulation of lipids on the pellicle surface, but virtually no substantial integration into the pellicle. Apparently, the structure of the 120 -min pellicle after initial rinsing with safflower oil was even of lower tenacity and loosened density in some cases. Several mechanisms may account for these observations. On the one hand, limited accumulation of the lipophilic molecules from the safflower oil in the pellicle layer is conceivable due to the high selectivity of pellicle formation, as indicated by the loosely adsorbed lipid micelles [Hannig and Joiner, 2006]. On the other hand, the abundant lipids of the safflower oil may even deplete relevant lipophilic molecules from the pellicle, impairing its protective properties [Hannig et al., 2012]. Taking together the present findings, the rinse with safflower oil certainly modulates the ultrastructure and the properties of the acquired pellicle, especially with respect to demineralization processes, but does not improve its protective properties. Interestingly, rinses with edible oils have also no impact on the initial bacterial colonization of enamel surfaces as observed in situ over $8 \mathrm{~h}$ [Hannig et al., 2012]. Thus, the relevance of edible oils in preventive dentistry seems to be limited with respect to dental erosion or caries, respectively.

Possibly other lipids with higher affinity to the pellicle structure are more appropriate than trigylcerides, mak- ing up the most relevant fraction of the safflower oil. Amphiphilic lipids such as phospholipids might be more suitable for sustainable integration into the pellicle structure and effective protective modification of pellicle ultrastructure [Slomiany et al., 1990; Hannig and Joiner, 2006; Hannig et al., 2012]. The protective properties could also be optimized by tanning agents such as polyphenols rather than by lipids [Joiner et al., 2003, 2004].

In the present study, erosive calcium and phosphate loss from enamel samples with an in situ formed pellicle depended predominantly on the $\mathrm{pH}$ of the acid. This is in line with the results of a previous study on erosive mineral loss from specimens without pellicle. This previous study was also based on photometric measurement of calcium and phosphate release at $\mathrm{pH}$ 2, 2.3 and 3 [Hannig et al., 2005]. During short-term exposure to solutions of $\mathrm{pH}$ 3 , the physiological in situ pellicle diminished erosive mineral loss clearly, but did not offer complete protection; at lower $\mathrm{pH}$, the protective effect is less pronounced. This corresponds to the findings of an in situ study based on the determination of microhardness of enamel slabs after consumption of different erosive beverages [Hannig et al., 2009]. Fast drinking of orange juice had no erosive effect, whereas Coke light and Sprite light caused a significant reduction of microhardness, meaning effective and comprehensive protection only against short and moderate erosive attacks due to pellicle and saliva [Hannig et al., 2009]. 
Generally, in contrast to previous postulates, the in situ pellicle layer has limited potential to protect the underlying enamel surface against erosion by hydrochloric acid or other erosive agents. The in situ formed pellicle acts mainly as an ion-permeable network, rather than a diffusion barrier [Hannig and Joiner, 2006]. Differing effects of the pellicle and of the modified pellicle on calcium and phosphate release were observed in the present study. The model for pellicle formation and for arrangement of calcium and phosphate ions at the tooth surface offers a possible explanation for the different behavior of calcium and phosphate ions (fig. 7) [Hannig and Joiner, 2006]. In the presence of saliva, the calcium ions of enamel have a more pronounced tendency than the phosphate ions to dissolve; this results in a double layer of ions at the enamel surface [Hay et al., 1982; Moreno et al., 1982; Hannig and Joiner, 2006]. The layer of calcium ions that are directly interacting with the pellicle precursor proteins of the basal layer is more prone to loss and might affect the solubility of the respective ions. Furthermore, the ionic radius of calcium $\left(\mathrm{Ca}^{2+}\right)$ amounts to $0.106 \mathrm{~nm}$, the negatively charged phosphate ions and hydrogen phosphate ions have a greater diameter. Besides the charge of the ions, their size might have an impact on the kinetics of demineralization and on the retention in the physiological as well as in the modified pellicle layer after application of safflower oil. This applies also for the interaction with the pellicle proteins and their functional groups at different $\mathrm{pH}$ values. A $\mathrm{pH}$-dependent modification of ion-binding pellicle proteins offers also an explanation for the unexpected, inhomogeneous effect of the $\mathrm{pH}$ on calcium and phosphate release through pellicles with and without application of oil. The application of the oil might modulate the interaction of the ions with these functional groups. This could be the reason for the increased phosphate release at $\mathrm{pH} 2.3$ following oil treatment.

\section{Conclusions}

(1) During short-term exposure to acidic agents, the physiological pellicle offers protection against erosive mineral loss in a $\mathrm{pH}$-dependent manner.

(2) The pellicle has to be regarded as a semi-permeable barrier.

(3) Rinsing with safflower oil modulates the ultrastructure of the pellicle and the passage of calcium and phosphate ions through the pellicle layer, but impairs protection against $\mathrm{HCl}$.

(4) Erosive processes at the tooth surface are rather complex and should be investigated with a combination of several methods at very short time intervals.

\section{Acknowledgment}

The study was supported by a grant from the DFG (Deutsche Forschungsgemeinschaft, HA 5192/2-1).

\section{Disclosure Statement}

The authors declare that they have no conflict of interest.

\section{References}

Asokan S: Oil pulling therapy. Indian J Dent Res 2008; 19:169.

Asokan S, Emmadi P, Chamundeswari R: Effect of oil pulling on plaque induced gingivitis: a randomized, controlled, triple-blind study. Indian J Dent Res 2009;20:47-51.

Asokan S, Rathinasamy TK, Inbamani N, Menon T, Kumar SS, Emmadi P, Raghuraman R: Mechanism of oil-pulling therapy - in vitro study. Indian J Dent Res 2011;22:34-37.

Attin T: Methods for assessment of dental erosion. Monogr Oral Sci 2006;20:152-172.

Attin T, Becker K, Hannig C, Buchalla W, Hilgers R: Method to detect minimal amounts of calcium dissolved in acidic solutions. Caries Res 2005a;39:432-436.
Attin T, Becker K, Hannig C, Buchalla W, Wiegand A: Suitability of a malachite green procedure to detect minimal amounts of phosphate dissolved in acidic solutions. Clin Oral Investig 2005b;9:203-207.

Buchalla W, Attin T, Roth P, Hellwig E: Influence of olive oil emulsions on dentin demineralization in vitro. Caries Res 2003;37:100-107.

Czernichow S, Thomas D, Bruckert E: N-6 fatty acids and cardiovascular health: a review of the evidence for dietary intake recommendations. Br J Nutr 2010;104:788-796.

Hannig C, Becker K, Häusler N, Hoth-Hannig W, Attin T, Hannig M: Protective effect of the in situ pellicle on dentin erosion - an ex vivo pilot study. Arch Oral Biol 2007a;52: 444-449.
Hannig C, Becker K, Yankeu-Ngalene VE, Attin T: Applicability of common methods for short time erosion analysis in vitro. Oral Health Prev Dent 2008;6:239-248.

Hannig C, Berndt D, Hoth-Hannig W, Hannig M: The effect of acidic beverages on the ultrastructure of the acquired pellicle: an in situ study. Arch Oral Biol 2009;54:518-526.

Hannig C, Hamkens A, Becker K, Attin R, Attin $\mathrm{T}$ : Erosive effects of different acids on bovine enamel: release of calcium and phosphate in vitro. Arch Oral Biol 2005;50:541-552.

Hannig C, Hannig M, Rehmer O, Braun G, Hellwig E, Al-Ahmad A: Fluorescence microscopic visualization and quantification of initial bacterial colonization on enamel in situ. Arch Oral Biol 2007b;52:1048-1056. 
Hannig C, Kirsch J, Al-Ahmad A, Kensche A, Hannig M, Kümmerer K: Do edible oils reduce bacterial colonization of enamel in situ? Clin Oral Investig 2012, E-pub ahead of print.

Hannig M: Ultrastructural investigation of pellicle morphogenesis at two different intraoral sites during a 24-h period. Clin Oral Investig 1999;3:88-95.

Hannig M: The protective nature of the salivary pellicle. Int Dent J 2002;52:417-423.

Hannig M, Balz M: Influence of in vivo formed salivary pellicle on enamel erosion. Caries Res 1999;33:372-379.

Hannig M, Balz M: Protective properties of salivary pellicles from two different intraoral sites on enamel erosion. Caries Res 2001;35: 142-148.

Hannig M, Hannig C: Nanomaterials in preventive dentistry. Nat Nanotechnol 2010;5:565569.

Hannig M, Hess NJ, Hoth-Hannig W, De Vrese M: Influence of salivary pellicle formation time on enamel demineralization - an in situ pilot study. Clin Oral Investig 2003;7:158161.

Hannig M, Joiner A: The structure, function and properties of the acquired pellicle. Monogr Oral Sci 2006;19:29-64.

Hara AT, Ando M, Gonzalez-Cabezas C, Cury JA, Serra MC, Zero DT: Protective effect of the dental pellicle against erosive challenges in situ. J Dent Res 2006;85:612-616.

Hay DI, Schluckebier SK, Moreno EC: Equilibrium dialysis and ultrafiltration studies of calcium and phosphate binding by human salivary proteins. Implications for salivary supersaturation with respect to calcium phosphate salts. Calcif Tissue Int 1982;34: 531-538.
Higa R, White V, Martinez N, Kurtz M, Capobianco E, Jawerbaum A: Safflower and olive oil dietary treatments rescue aberrant embryonic arachidonic acid and nitric oxide metabolism and prevent diabetic embryopathy in rats. Mol Hum Reprod 2010;16:286-295.

Jaeggi T, Lussi A: Prevalence, incidence and distribution of erosion. Monogr Oral Sci 2006; 20:44-65.

Joiner A, Muller D, Elofsson UM, Arnebrant T: Ellipsometry analysis of the in vitro adsorption of tea polyphenols onto salivary pellicles. Eur J Oral Sci 2004;112:510-515.

Joiner A, Muller D, Elofsson UM, Malmsten M, Arnebrant T: Adsorption from black tea and red wine onto in vitro salivary pellicles studied by ellipsometry. Eur J Oral Sci 2003;111: 417-422.

Krist S, Buchbauer G, Klausberger C: Lexikon der pflanzlichen Fette und Öle. Wien, Springer, 2008.

Larsen MJ, Poulsen S, Hansen I: Erosion of the teeth: prevalence and distribution in a group of Danish school children. Eur J Paediatr Dent 2005;6:44-47.

Lussi A, Hellwig E, Zero D, Jaeggi T: Erosive tooth wear: diagnosis, risk factors and prevention. Am J Dent 2006;19:319-325.

Lussi A, Jaeggi T, Zero D: The role of diet in the aetiology of dental erosion. Caries Res 2004; 38(suppl 1):34-44.

Magalhaes AC, Rios D, Moino AL, Wiegand A, Attin T, Buzalaf MA: Effect of different concentrations of fluoride in dentifrices on dentin erosion subjected or not to abrasion in situ/ex vivo. Caries Res 2008;42:112-116.
Moreno EC, Kresak M, Hay DI: Adsorption thermodynamics of acidic proline-rich human salivary proteins onto calcium apatites. J Biol Chem 1982;257:2981-2989.

Reynolds EC: Anticariogenic complexes of amorphous calcium phosphate stabilized by casein phosphopeptides: a review. Spec Care Dentist 1998;18:8-16.

Schüpbach P, Oppenheim FG, Lendenmann U, Lamkin MS, Yao Y, Guggenheim B: Electron-microscopic demonstration of prolinerich proteins, statherin, and histatins in acquired enamel pellicles in vitro. Eur J Oral Sci 2001;109:60-68.

Slomiany BL, Murty VL, Mandel ID, Sengupta S, Slomiany A: Effect of lipids on the lactic acid retardation capacity of tooth enamel and cementum pellicles formed in vitro from saliva of caries-resistant and caries-susceptible human adults. Arch Oral Biol 1990;35:175-180.

Slomiany BL, Murty VL, Zdebska E, Slomiany A, Gwozdzinski K, Mandel ID: Tooth surfacepellicle lipids and their role in the protection of dental enamel against lactic-acid diffusion in man. Arch Oral Biol 1986;31:187-191.

Suzuki K, Tsubaki S, Fujita M, Koyama N, Takahashi M, Takazawa K: Effects of safflower seed extract on arterial stiffness. Vasc Health Risk Manag 2010;6:1007-1014.

Wegehaupt F, Gunthart N, Sener B, Attin T: Prevention of erosive/abrasive enamel wear due to orange juice modified with dietary supplements. Oral Dis 2011;17:508-514.

Wiegand A, Gutsche M, Attin T: Effect of olive oil and an olive-oil-containing fluoridated mouthrinse on enamel and dentin erosion in vitro. Acta Odontol Scand 2007;65:357-361. 